\title{
Robust Joint Optimization of Non-regenerative MIMO Relay Channels with Imperfect CSI
}

\author{
Ebrahim Avazkonandeh Gharavol and Erik G. Larsson
}

\section{Linköping University Post Print}

N.B.: When citing this work, cite the original article.

C2011 IEEE. Personal use of this material is permitted. However, permission to reprint/republish this material for advertising or promotional purposes or for creating new collective works for resale or redistribution to servers or lists, or to reuse any copyrighted component of this work in other works must be obtained from the IEEE.

Ebrahim Avazkonandeh Gharavol and Erik G. Larsson, Robust Joint Optimization of Nonregenerative MIMO Relay Channels with Imperfect CSI, 2011, Proceedings of the 45th Annual Asilomar Conference on Signals, Systems, and Computers.

Postprint available at: Linköping University Electronic Press

http://urn.kb.se/resolve?urn=urn:nbn:se:liu:diva-70934 


\title{
Robust Joint Optimization of Nonregenerative MIMO Relay Channels with Imperfect CSI
}

\author{
Ebrahim A. Gharavol and Erik G. Larsson \\ Division of Communication Systems, \\ Electrical Engineering Department (ISY) \\ Linköping University, 58183 Linköping, Sweden
}

\begin{abstract}
In this paper, we deal with the problem of joint optimization of the source precoder, the relay beamformer and the destination equalizer in a nonregenerative relay network with only a partial knowledge of the Channel State Information (CSI). We model the partial CSI using a deterministic norm bounded error model, and we use a system-wide mean square error performance measure which is constrained based on the transmit power regulations for both source and relay nodes. Most conventional designs employ the average performance optimization, however, we solve this problem from a worst-case design perspective. The original problem formulation is a semi-infinite trilinear optimization problem which is not convex. To solve this problem we extend the existing theories to deal with the constraints which are semi-infinite in different independent complex matrix variables. We show that the equivalent approximate problem is a set of linear matrix inequalities, that can be solved iteratively. Finally simulation results assess the performance of the proposed scheme.
\end{abstract}

\section{INTRODUCTION}

Relay networks are known to effectively expand the coverage area and increase the signal strength of a typical communication system. The relay channels are considered in the recent wireless communication standards like Long Term Evolution (LTE), LTE-Advanced (LTE-A), and WiMAX. To best exploit the diversity of such a system equipped with multiple antennas, beamforming process is a promising method. To implement the beamforming process, the Channel State Information (CSI) plays a dominant role, and therefore it is conventionally assumed that the CSI is perfectly known at both the transmit and the receive stations. However, this assumption is not a practical one, due to the erroneous channel estimations and the limited feedback mechanism between the receive and the transmit sides. It is not practically possible to acquire perfect CSI and dispatch it to the network nodes.

In [1]-[3] the problem of the robust beamforming is studied for different network configurations and with different assumptions. These papers are mostly concerning MISO broadcast channels. In [4]-[6] the problem of beamforming in half duplex relay channels are considered. Distributed relay beamforming

This paper describes work undertaken in the context of the LOLA project - Achieving LOw-LAtency in Wireless Communications (www.ict-lola.eu). The research leading to these results has received funding from the European Communitys Seventh Framework Program under grant agreement No. 248993. E. Larsson is a Royal Swedish Academy of Sciences (KVA) Research Fellow supported by a grant from the Knut and Alice Wallenberg Foundation. is considered in [4] and three different half-duplex beamforming problems are formulated and subsequently their solutions are provided in terms of closed form solutions or via convex optimization. The concept of general rank beamforming is introduced in [5] to solve the beamforming problem in a relay network with multiple antennas employed only in the relay node. Soft and hard interference cancellation methods which are important for all types of relay networks are presented in [6]. This work shows that the subspace computation and the generalized waterfilling methods are essential in the relay beamforming.

The problem of robust beamforming is considered in [7][11]. In [7], the problem of robust beamforming is solved for single antenna transmit and receive stations with multiple single antenna relay nodes. The CSI is assumed to be norm bounded, and it is shown that the robust solution is a Semidefinite Programming (SDP) problem. Zero forcing and Minimum Mean Square Error (MMSE) based beamforming for relay assisted downlink transmission is considered in [8]. In [9] the problem of beamforming for a Multiple Input Multiple Output (MIMO) relay channel is studied. In this work it is assumed that there is no direct path between the source and the destination and it is also assumed that the relay node perfectly knows the CSI of the channel between the source and the relay. It is also shown that the robust solution is a SDP. An MMSE based robust relay design for the relay channel is introduced in [10]. In this paper it is assumed that the CSI is impaired by Gaussian noise and an average performance measure optimization solution based on the quadratic matrix programming is proposed. Finally [11] extends the general rank precoding design to the case with uncertain CSI.

In this paper we consider a half-duplex relay with multiple antennas at the source, the relay node and the destination. The CSI is impaired with a norm bounded error matrix, and we assume that there is a direct link between the source and the relay station. We use a worst case analysis basis to study the system. We extend the current tools to be applicable to constraints with more that one uncertain variables. The robust solution finally would be a set of Linear Matrix Inequalities (LMI's) here. Our work is a direct successor of [7] and [9] and generalize them in different directions.

In this paper the following notations are used: Boldfaced letters are used to show vectors or the matrices while scalar 


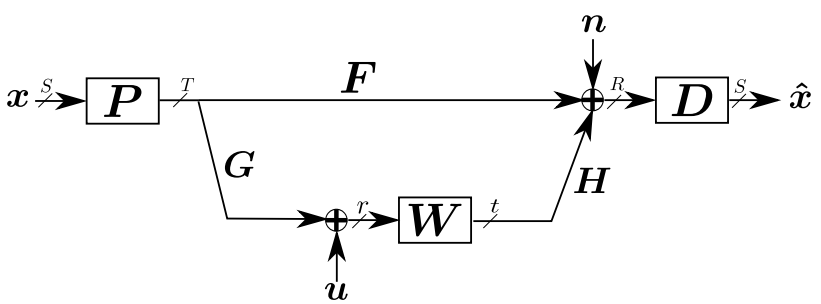

Fig. 1. OWRC Signal Flow Graph

variables or constants are shown with no specific formatting. The field of the complex numbers, the field of the $n$ dimensional complex-valued vector spaces and the field of the $m \times n$ complex-valued matrices are denoted using $\mathbb{C}$, $\mathbb{C}^{n}$ and $\mathbb{C}^{m \times n}$, respectively. For any vector $\boldsymbol{x}$ or for any matrix $\boldsymbol{X},\|\boldsymbol{x}\|$ and $\|\boldsymbol{X}\|_{F}$ denote the Euclidean and the Frobenius norms of that vector or matrix, respectively. The positive semidefinite matrix $\boldsymbol{X}$ is denoted using $\boldsymbol{X} \succeq 0$. To show a vertically concatenated vectorized version of a matrix, vec [], to show a vertical concatenation of a set of matrices to build a taller block matrix, MAT [], and to show the block diagonal concatenation of a set of matrices blkdiag [...] are used, respectively. Finally, $\mathrm{E}_{\boldsymbol{x}}[f(\boldsymbol{x})]$ is the mathematical expectation of $f(\boldsymbol{x})$ with respect to the stochastic variable $\boldsymbol{x}$.

\section{System Model and Problem Formulation}

A wireless relay system in which the source, the relay and the destination nodes are equipped with multiple antennas is depicted in Fig. 1. In this system, to initiate a half-duplex communication service, two distinct time slots are required. In the first time slot, the source node broadcasts its data to reach to both the relay and the destination nodes, and in the second slot, the relay node transmits the amplified signal towards the destination. By means of this relaying process, the Signal to Noise Ratio (SNR) at the destination is improved and thus the destination node is helped to better decode its received signal. To transmit $S$ independent streams of zero-mean, unitvariance data $\left(\boldsymbol{x} \in \mathbb{C}^{T}\right)$ with independent elements toward the destination, the source node is equipped with $T$ transmit antennas. The destination is equipped with $R$ receive antennas, while the relay has $r$ and $t$ receive and transmit antennas, respectively. To better compensate the fading channel, the source node precodes the data using a precoding matrix $\boldsymbol{P} \in \mathbb{C}^{T \times S}$. The precoded data is sent over two wireless fading channels, namely $\boldsymbol{F} \in \mathbb{C}^{R \times T}$ and $\boldsymbol{G} \in \mathbb{C}^{r \times T}$. At the relay node, the received signal is amplified using $\boldsymbol{W} \in \mathbb{C}^{t \times r}$ and the resultant signal is transmitted to the destination in the next time slot over the wireless channel $\boldsymbol{H} \in \mathbb{C}^{R \times t}$. At the destination, after appropriately combining the received signals and decoding using a linear decoder $\boldsymbol{D} \in \mathbb{C}^{S \times R}$, the received signal is as follows:

$$
\hat{\boldsymbol{x}}=(\boldsymbol{D F P}+\boldsymbol{D H W G P}) \boldsymbol{x}+\boldsymbol{D H W u}+\boldsymbol{D} \boldsymbol{n},
$$

where $\boldsymbol{u} \in \mathbb{C}^{r}$ and $\boldsymbol{n} \in \mathbb{C}^{R}$ are the additive zero-mean noise signals with independent elements and $\sigma_{u}^{2}$ and $\sigma_{n}^{2}$ variances, respectively. Due to the limited feedback between the nodes, it is assumed that the nominal value of the CSI is known to the system. In other words, the CSI follows the norm bounded error model:

$$
\begin{aligned}
& \boldsymbol{F} \in \mathcal{F}=\left\{\tilde{\boldsymbol{F}}+\boldsymbol{\Delta}_{F} \mid\left\|\boldsymbol{\Delta}_{F}\right\|_{F} \leq \delta_{F}\right\}, \\
& \boldsymbol{G} \in \mathcal{G}=\left\{\tilde{\boldsymbol{G}}+\boldsymbol{\Delta}_{G} \mid\left\|\boldsymbol{\Delta}_{G}\right\|_{F} \leq \delta_{G}\right\}, \\
& \boldsymbol{H} \in \mathcal{H}=\left\{\tilde{\boldsymbol{H}}+\boldsymbol{\Delta}_{H} \mid\left\|\boldsymbol{\Delta}_{H}\right\|_{F} \leq \delta_{H}\right\} .
\end{aligned}
$$

where $\tilde{\boldsymbol{F}}, \tilde{\boldsymbol{G}}$, and $\tilde{\boldsymbol{H}}$ are the fixed nominal value of the CSI for each of the channels and $\boldsymbol{\Delta}_{F}, \boldsymbol{\Delta}_{G}$, and $\boldsymbol{\Delta}_{H}$, are the random norm-bounded variations (uncertainties) around these nominal values.

In this paper, our goal is to jointly optimize the source precoder, the relay beamformer, and the destination equalizer. To do so, we use the system-wide MSE as the performance measure of the system, and we restrict the optimization problem with the power budgets of both the source and the relay nodes. By employing a worst-case design approach, the optimal solutions will be valid for all the realizations of the CSI that satisfy (2). To facilitate the computation of the system-wide MSE, and the transmit powers of the source and the relay nodes, we use the following lemma.

Lemma 1: For any set of zero-mean, independent and identically distributed random vectors with independent elements and individual variances of $\mathrm{E}_{\boldsymbol{x}_{i}}\left[\boldsymbol{x}_{i}^{*} \boldsymbol{x}_{i}\right]=\sigma_{i}^{2}$ we have

$$
\mathrm{E}_{\left\{\boldsymbol{x}_{i}\right\}_{i}}\left[\left\|\sum_{i} \boldsymbol{A}_{i} \boldsymbol{x}_{i}\right\|^{2}\right]=\sum_{i} \sigma_{i}^{2}\left\|\boldsymbol{A}_{i}\right\|_{F}^{2}
$$

Proof: It is clear that

$$
\begin{aligned}
\mathrm{E}_{\left\{\boldsymbol{x}_{i}\right\}_{i}}\left[\left\|\sum_{i} \boldsymbol{A}_{i} \boldsymbol{x}_{i}\right\|^{2}\right] & =\sum_{i} \sum_{j} \mathrm{E}_{\boldsymbol{x}_{i}, \boldsymbol{x}_{j}}\left[\boldsymbol{x}_{i}^{*} \boldsymbol{A}_{i}^{*} \boldsymbol{A}_{j} \boldsymbol{x}_{j}\right] \\
& =\sum_{i} \sum_{j} \mathrm{E}_{\boldsymbol{x}_{i}, \boldsymbol{x}_{j}}\left[\operatorname{tr}\left[\boldsymbol{x}_{i}^{*} \boldsymbol{A}_{i}^{*} \boldsymbol{A}_{j} \boldsymbol{x}_{j}\right]\right] \\
& =\sum_{i} \sum_{j} \mathrm{E}_{\boldsymbol{x}_{i}, \boldsymbol{x}_{j}}\left[\operatorname{tr}\left[\boldsymbol{A}_{i}^{*} \boldsymbol{A}_{j} \boldsymbol{x}_{j} \boldsymbol{x}_{i}^{*}\right]\right] \\
& =\sum_{i} \sum_{j} \operatorname{tr}\left[\boldsymbol{A}_{i}^{*} \boldsymbol{A}_{j} \mathrm{E}_{\boldsymbol{x}_{i}, \boldsymbol{x}_{j}}\left[\boldsymbol{x}_{j} \boldsymbol{x}_{i}^{*}\right]\right]
\end{aligned}
$$

By plugging the following

$$
\mathrm{E}_{\boldsymbol{x}_{i}, \boldsymbol{x}_{j}}\left[\boldsymbol{x}_{i} \boldsymbol{x}_{j}^{*}\right]= \begin{cases}\sigma_{i}^{2} \boldsymbol{I} & i=j, \\ \mathbf{0} & i \neq j,\end{cases}
$$

into the expectation, we get

$$
\mathrm{E}_{\left\{\boldsymbol{x}_{i}\right\}_{i}}\left[\left\|\sum_{i} \boldsymbol{A}_{i} \boldsymbol{x}_{i}\right\|^{2}\right]=\sum_{i} \sigma_{i}^{2} \operatorname{tr}\left[\boldsymbol{A}_{i}^{*} \boldsymbol{A}_{i}\right]
$$

which proves the lemma.

Based on this Lemma 1, the system wide MSE (MSE), and the transmit powers of the source $\left(\mathrm{TxP}_{s}\right)$ and the relay $\left(\mathrm{TxP}_{r}\right)$ 
nodes are defined as follows:

$$
\begin{aligned}
\mathrm{MSE} \triangleq & \mathrm{E}_{\boldsymbol{x}, \boldsymbol{u}, \boldsymbol{n}}\left[\|\hat{\boldsymbol{x}}-\boldsymbol{x}\|^{2}\right] \\
= & \|\boldsymbol{D} \boldsymbol{F P}+\boldsymbol{D} \boldsymbol{H} \boldsymbol{W} \boldsymbol{G P}-\boldsymbol{I}\|_{F}^{2}+\sigma_{u}^{2}\|\boldsymbol{D} \boldsymbol{H} \boldsymbol{W}\|_{F}^{2} \\
& +\sigma_{n}^{2}\|\boldsymbol{D}\|_{F}^{2} \\
\mathrm{TxP}_{s} \triangleq & \mathrm{E}_{\boldsymbol{x}}\left[\|\boldsymbol{P} \boldsymbol{x}\|_{2}^{2}\right] \\
= & \|\boldsymbol{P}\|_{F}^{2} \\
\mathrm{TxP}_{r} \triangleq & \mathrm{E}_{\boldsymbol{x}, \boldsymbol{u}}\left[\|\boldsymbol{W} \boldsymbol{G P} \boldsymbol{x}+\boldsymbol{W} \boldsymbol{u}\|_{2}^{2}\right] \\
= & \|\boldsymbol{W} \boldsymbol{G P}\|_{F}^{2}+\sigma_{u}^{2}\|\boldsymbol{W}\|_{F}^{2}
\end{aligned}
$$

Remark 1: It is clear that the MSE is a multilinear function of the design variables, while $\operatorname{TxP}_{s}$ and $\operatorname{TxP}_{r}$ are convex (linear) and biconvex functions in the same variables, respectively.

Using these quantities, the problem formulation in its epigraph form will be,

$$
\begin{array}{cll}
\underset{\boldsymbol{P}, \boldsymbol{W}, \boldsymbol{D}, \tau \geq 0}{\operatorname{minimize}} & \tau \\
\text { subject to } & \operatorname{TxP}_{s} \leq P_{s} & \\
& \operatorname{TxP}_{r} \leq P_{r}, \quad \forall \boldsymbol{G} \in \mathcal{G} \\
& \operatorname{MSE} \leq \tau, \quad \forall \boldsymbol{F} \in \mathcal{F}, \boldsymbol{G} \in \mathcal{G}, \boldsymbol{H} \in \mathcal{H}
\end{array}
$$

where $P_{s}$ and $P_{r}$ are the power limits of the source and the relay nodes.

Remark 2: It is clear that in this robust problem formulation, the last constraints are semi-infinite constraints, i.e., they have infinitely many realizations, while the constraint functions are not simultaneously convex in the design variables. Both these features make the proposed problem very hard to solve (a NP-hard problem). Additionally, it should be mentioned that the last constraint is threefold semi-infinite (in three different and independent variables).

Remark 3: Clearly if we set $\delta_{F}=\delta_{G}=\delta_{H}=0, \mathcal{F}, \mathcal{G}$ and $\mathcal{H}$ would become singleton sets, and these sets are reduced to the nominal values of the CSI corresponding to each channel. This case is the perfect CSI scenario which is studied deeply in the literature [4]-[6]. We will use this scenario as a benchmark to compare the performance of the proposed algorithm here. $\checkmark$

In the following section we choose a two stage mechanism to simplify the problem (7) to an equivalent problem, and then we provide an iterative algorithm which solves the equivalent problem optimally.

\section{SOLUTION}

In this section, we deal with the nonconvex problem (7) and convert it into a convex equivalent problem, for which computationally efficient interior point methods exist. To do so, we employ a two stage process: first, we deal with the semi-infiniteness of the last two constraints of (7) using the generalized version of Petersen's lemma for complex valued matrices, and then we propose an iterative algorithm based on the ACS, to suboptimally solve the multilinear (nonconvex) problem. We start with the last constraint which, based on Remark 2, is multilinear in the design variables and threefold semi-infinite. The $\operatorname{TxP}_{r}$ constraint needs a similar procedure which is not repeated here. To deal with the MSE constraint, first using $\left\|\boldsymbol{A}_{i}\right\|_{F}=\left\|\operatorname{vec}\left[\boldsymbol{A}_{i}\right]\right\|_{2}$, the MSE is recast as follows:

$$
\text { MSE }=\left\|\left[\begin{array}{c}
\operatorname{vec}[\boldsymbol{D} \boldsymbol{F} \boldsymbol{P}]+\operatorname{vec}[\boldsymbol{D H} \boldsymbol{W} \boldsymbol{G P}]-\operatorname{vec}[\boldsymbol{I}]] \\
\sigma_{u} \operatorname{vec}[\boldsymbol{D} \boldsymbol{H} \boldsymbol{W}] \\
\sigma_{n} \operatorname{vec}[\boldsymbol{D}]
\end{array}\right]\right\|^{2}
$$

After inserting the form of (2) into the above equation, and neglecting the higher order uncertainty terms ${ }^{1}$, it is possible to recast the MSE as MSE $\triangleq\|\boldsymbol{\mu}\|^{2}$ where

$\boldsymbol{\mu}=\tilde{\boldsymbol{\mu}}+\boldsymbol{M}_{F} \operatorname{vec}\left[\boldsymbol{\Delta}_{F}\right]+\boldsymbol{M}_{G} \operatorname{vec}\left[\boldsymbol{\Delta}_{G}\right]+\boldsymbol{M}_{H} \operatorname{vec}\left[\boldsymbol{\Delta}_{H}\right]$,

where

$$
\tilde{\boldsymbol{\mu}}=\left[\begin{array}{c}
\operatorname{vec}[\boldsymbol{D} \tilde{\boldsymbol{F}} \boldsymbol{P}]+\operatorname{vec}[\boldsymbol{D} \tilde{\boldsymbol{H}} \boldsymbol{W} \tilde{\boldsymbol{G}} \boldsymbol{P}]-\operatorname{vec}[\boldsymbol{I}] \\
\sigma_{u} \operatorname{vec}[\boldsymbol{D} \tilde{\boldsymbol{H}} \boldsymbol{W}] \\
\sigma_{n} \operatorname{vec}[\boldsymbol{D}]
\end{array}\right]
$$

and subsequently

$$
\begin{aligned}
& \boldsymbol{M}_{F}=\left[\begin{array}{c}
\boldsymbol{P}^{T} \otimes \boldsymbol{D} \\
\mathbf{0}
\end{array}\right] \in \mathbb{C}^{S^{\prime} \times R T}, \\
& \boldsymbol{M}_{G}=\left[\begin{array}{c}
\boldsymbol{P}^{T} \otimes \boldsymbol{D} \tilde{\boldsymbol{H}} \boldsymbol{W} \\
\mathbf{0}
\end{array}\right] \in \mathbb{C}^{S^{\prime} \times r T}, \\
& \boldsymbol{M}_{H}=\left[\begin{array}{c}
(\boldsymbol{W} \tilde{\boldsymbol{G}} \boldsymbol{P})^{T} \otimes \boldsymbol{D} \\
\sigma_{u} \boldsymbol{W}^{T} \otimes \boldsymbol{D} \\
\mathbf{0}
\end{array}\right] \in \mathbb{C}^{S^{\prime} \times R t},
\end{aligned}
$$

where $S^{\prime}=S(S+R+r)$. Using the Schur complement lemma [12], the MSE constraint can be recast as the following LMI:

$$
\left[\begin{array}{cc}
\tau & \tilde{\boldsymbol{\mu}}^{*} \\
\tilde{\boldsymbol{\mu}} & \boldsymbol{I}
\end{array}\right] \succeq-\sum_{\zeta \in\{F, G, H\}}\left[\begin{array}{cc}
0 & \left(\boldsymbol{M}_{\zeta} \operatorname{vec}\left[\boldsymbol{\Delta}_{\zeta}\right]\right)^{*} \\
\boldsymbol{M}_{\zeta} \operatorname{vec}\left[\boldsymbol{\Delta}_{\zeta}\right] & \mathbf{0}
\end{array}\right]
$$

To proceed with this constraint, we formally generalize the Petersen's Lemma to multiple complex valued uncertainties [13]-[14]. It is noteworthy that the complex valued version of this lemma for a single uncertainty is proved in [15].

Lemma 2: Given matrices $\boldsymbol{A}$ and $\left\{\boldsymbol{P}_{i}, \boldsymbol{Q}_{i}\right\}_{i=1}^{N}$ with $\boldsymbol{A}=$ $\boldsymbol{A}^{*}$, the semi-infinite LMI of the form of

$$
\boldsymbol{A} \succeq \sum_{i=1}^{N}\left(\boldsymbol{P}_{i}^{*} \boldsymbol{X}_{i} \boldsymbol{Q}_{i}+\boldsymbol{Q}_{i}^{*} \boldsymbol{X}_{i}^{*} \boldsymbol{P}_{i}\right), \quad \forall i, \boldsymbol{X}_{i}:\left\|\boldsymbol{X}_{i}\right\| \leq \varkappa_{i} ;
$$

holds if and only if there exist nonnegative real numbers $\epsilon_{1}, \cdots, \epsilon_{N}$ such that

$$
\left[\begin{array}{cc}
\boldsymbol{A}-\sum_{i=1}^{N} \epsilon_{i} \boldsymbol{Q}_{i}^{*} \boldsymbol{Q}_{i} & \text { MAT }\left[\left\{-\varkappa_{i} \boldsymbol{P}_{i}\right\}_{i=1}^{N}\right]^{*} \\
\operatorname{MAT}\left[\left\{-\varkappa_{i} \boldsymbol{P}_{i}\right\}_{i=1}^{N}\right] & \text { blkdiag }\left[\left\{\epsilon_{i} \boldsymbol{I}\right\}_{i=1}^{N}\right]
\end{array}\right] \succeq 0 .
$$

Proof: The proof is omitted due to length constraints.

\footnotetext{
${ }^{1} \boldsymbol{D} \boldsymbol{\Delta}_{H} \boldsymbol{W} \boldsymbol{\Delta}_{G} \boldsymbol{P}$ has a very small norm relative to the other terms and introduces a nonlinearity to the system which makes it mathematically intractable.
} 
Using Lemma 2, and by appropriately choosing its parameters as follows,

$$
\begin{aligned}
\boldsymbol{A} & =\left[\begin{array}{cc}
\tau & \tilde{\boldsymbol{\mu}}^{*} \\
\tilde{\boldsymbol{\mu}} & \boldsymbol{I}
\end{array}\right] \in \mathbb{C}^{\left(1+S^{\prime}\right) \times\left(1+S^{\prime}\right)} \\
\boldsymbol{Q}_{1} & =\boldsymbol{Q}_{2}=\boldsymbol{Q}_{3}=\left[\begin{array}{ll}
-1 & \mathbf{0}^{T}
\end{array}\right] \in \mathbb{C}^{1 \times\left(1+S^{\prime}\right)} \\
\boldsymbol{P}_{1} & =\left[\begin{array}{ll}
\mathbf{0} & \boldsymbol{M}_{F}^{*}
\end{array}\right] \in \mathbb{C}^{R T \times\left(1+S^{\prime}\right)}, \\
\boldsymbol{P}_{2} & =\left[\begin{array}{ll}
\mathbf{0} & \boldsymbol{M}_{G}^{*}
\end{array}\right] \in \mathbb{C}^{r T \times\left(1+S^{\prime}\right)}, \\
\boldsymbol{P}_{3} & =\left[\begin{array}{ll}
\mathbf{0} & \boldsymbol{M}_{H}^{*}
\end{array}\right] \in \mathbb{C}^{R t \times\left(1+S^{\prime}\right)}, \\
\boldsymbol{X}_{1} & =\operatorname{vec}\left[\boldsymbol{\Delta}_{F}\right], \boldsymbol{X}_{1}=\operatorname{vec}\left[\boldsymbol{\Delta}_{G}\right], \boldsymbol{X}_{3}=\operatorname{vec}\left[\boldsymbol{\Delta}_{H}\right],
\end{aligned}
$$

It is possible to rewrite the MSE constraint as the following finite(single) LMIs:

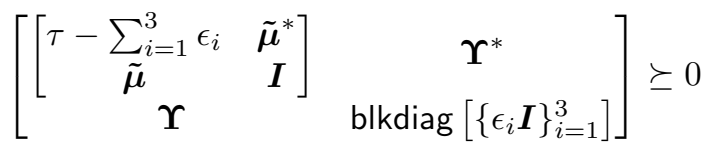

$$
\begin{aligned}
& \operatorname{diag}\left[\tau, \epsilon_{1}, \epsilon_{2}, \epsilon_{3}\right] \succeq 0
\end{aligned}
$$

where

$$
\boldsymbol{\Upsilon}=\left[\begin{array}{lll}
-\delta_{F} \boldsymbol{P}_{1}^{T} & -\delta_{G} \boldsymbol{P}_{2}^{T} & -\delta_{H} \boldsymbol{P}_{3}^{T}
\end{array}\right]^{T} .
$$

Using a similar procedure for the other semi-infinite constraint, i.e., the $\operatorname{TxP}_{r}$ constraint, it is possible to show that $\mathrm{TxP}_{r}=\|\boldsymbol{\pi}\|^{2}$, where

$$
\begin{aligned}
\boldsymbol{\pi} & =\tilde{\boldsymbol{\pi}}+\boldsymbol{P}_{G} \operatorname{vec}\left[\boldsymbol{\Delta}_{G}\right], \\
\tilde{\boldsymbol{\pi}} & =\left[\begin{array}{ll}
\operatorname{vec}[\boldsymbol{W} \tilde{\boldsymbol{G}} \boldsymbol{P}]^{T} & \sigma_{u} \operatorname{vec}[\boldsymbol{W}]^{T}
\end{array}\right]^{T}, \\
\boldsymbol{P}_{G} & =\left[\begin{array}{ll}
\left(\boldsymbol{P}^{T} \otimes \boldsymbol{W}\right)^{T} & \mathbf{0}
\end{array}\right]^{T} .
\end{aligned}
$$

Similarly, it is possible to replace this constraint with the following single LMI:

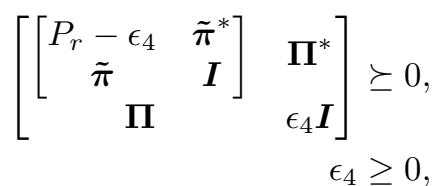

where

$$
\boldsymbol{\Pi}=\left[\begin{array}{lll}
\mathbf{0} & -\delta_{G} & \boldsymbol{P}_{G}^{*}
\end{array}\right] .
$$

Putting all these equivalent constraints together will result in the following LMI which replaces (7):

$$
\begin{aligned}
& \underset{P, W, D, \tau}{\operatorname{minimize}} \tau \\
& \text { subject to }\|\boldsymbol{P}\|_{F}^{2} \leq P_{s} \\
& \operatorname{diag}\left[\tau, \epsilon_{1}, \epsilon_{2}, \epsilon_{3}, \epsilon_{4}\right] \succeq 0
\end{aligned}
$$

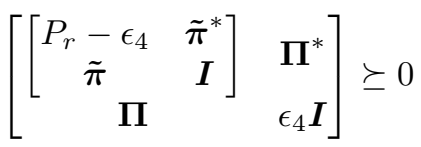

$$
\begin{aligned}
& {\left[\begin{array}{cc}
{\left[\begin{array}{cc}
\tau-\sum_{\tilde{i}=1}^{3} \epsilon_{i} & \tilde{\boldsymbol{\mu}}^{*} \\
\tilde{\boldsymbol{\mu}} & \boldsymbol{I}
\end{array}\right]} & \text { blkdiag }\left[\left\{\epsilon_{i} \boldsymbol{I}\right\}_{i=1}^{3}\right]
\end{array}\right] \succeq 0}
\end{aligned}
$$

The above problem is not a semi-infinite problem, but it is still nonconvex. Due to biconvex and multilinear structure of the elements of $\Pi$ and $\Upsilon$, we resort to an iterative algorithms derived based on the Alternating Convex Search (ACS) method, i.e., Algorithm 1.

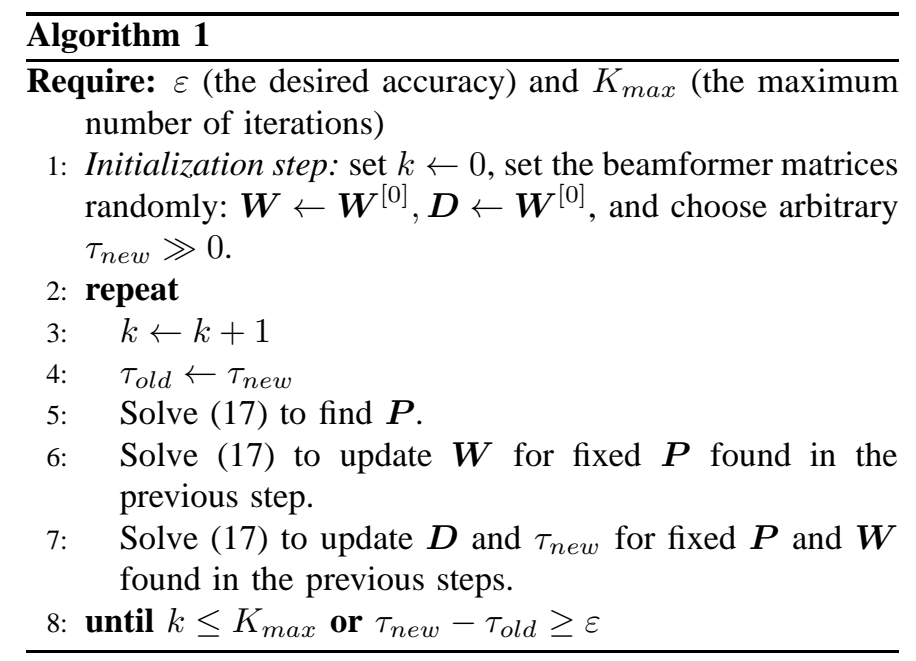

The convergence of the above algorithm is inherent from the ACS method. Since the original problem is not a convex problem, we may have different solutions due to different initial matrices. It is easily possible to show that if $\delta_{F}=$ $\delta_{G}=\delta_{H}=0$ the above problem reduces to problem with full (perfect) CSI. In that case, (17) becomes a simple SOCP as follows:

$$
\begin{aligned}
\underset{\boldsymbol{P}, \boldsymbol{W}, \boldsymbol{D}, \tau}{\operatorname{minimize}} & \tau \\
\text { subject to } & \|\boldsymbol{P}\|_{F}^{2} \leq P_{s} \\
& \|\tilde{\boldsymbol{\pi}}\|^{2} \leq P_{r} \\
& \|\tilde{\boldsymbol{\mu}}\|^{2} \leq \tau
\end{aligned}
$$

\section{Simulation Results}

To assess the performance of the proposed algorithm, the following simulation is done, and the results are summarized here in this section. The simulation setup is as follows: the system is used to transfer $S=2$ streams of independent data between the source and the destination. The number of transmit and receive antennas in the source, relay and destination are equal to $T=R=t=r=4$. Both source and relay power budgets are set to be equal to $P_{r}=P_{s}=1$. The convergence parameters of the algorithm are set to $K_{\max }=1000$, and $\varepsilon=10^{-4}$. The initial value of the relay precoder and the destination equalizer matrices are set to be equal to zero. The set of channels are generated randomly to model Rayleigh fading channels. In Fig. 2 and Fig. 3 the system-wide MSE and the transmit power of the relay node for a single typical run are depicted.

In Fig. 2 the MSE of the relay system is depicted. As can be seen, the MSE increases proportionally with the increase of the uncertainty size. It is expected since $\boldsymbol{\mu}$ is a linear combination of the uncertainty matrices, and the uncertainty 


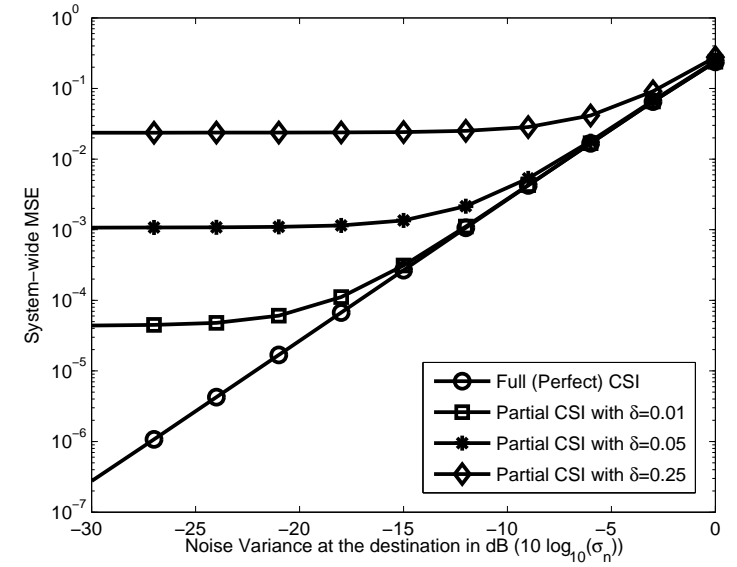

Fig. 2. System-wide MSE

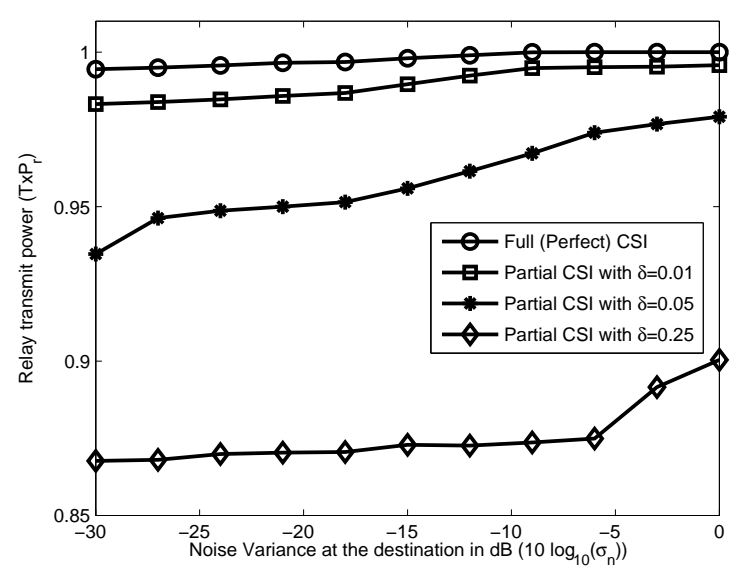

Fig. 3. Relay Transmit Power

size is the norm of these matrices. For smaller noise powers, the MSE is mostly dominated by the uncertainty terms rather than the noise terms, and because of that the MSE is more or less constant with respect to the noise power. However, for higher noise powers, the MSE is dominated by the noise terms and on the rightmost part, the MSE is only a function of the noise power but not the uncertainty size. Since the MSE is proportional to the uncertainty size, the perfect (full) CSI case outperforms the other cases with uncertainty, because the full CSI case is a special case of the partial CSI case with $\delta=0$.

In Fig. 3 the transmit power of the relay station is depicted. Unlike the source transmit power constraint which is satisfied with the equality sign, the relay transmit power constraint is not satisfied with the equality sign to possibly prevent the over amplification of the relay noise power to the destination. As can be seen with the increase of the noise power, and to maintain a minimum MSE, the transmit power of the relay station increases. It is clear that the transmit power is also a function of the uncertainty size. With the increase of the uncertainty size, the transmit power decreases. It is because that the MSE increases with the increase of the $\delta$, and again to maintain the minimum MSE, the transmit power should be decreased.

\section{CONClusion}

In this paper the problem of the robust joint optimization of a one-way relay channel is studied. The system-wide MSEbased problem formulation which is nonconvex in nature, is considered. An approximate convex solution for this problem is proposed here. Simulation results of the solution show that the MSE of the system increases with the increase of the uncertainty size of the CSI. For the smaller noise powers, the MSE is dominated by the uncertainty terms while for the larger noise powers MSE is dominated by the noise terms. Also, It can be seen that to prevent the amplification of the relay station noise in the destination, the relay transmitter power is less than the actual power limit. It can be concluded as well that the transmit power of the relay station depends on the uncertainty size: the larger the uncertainty size, the less the transmit power.

\section{REFERENCES}

[1] S. Shahbazpanahi, A.B. Gershman, Z.-Q. Luo, and K.M. Wong, "Robust adaptive beamforming for general-rank signal models," IEEE Tran. Signal Processing, vol. 51, no. 9, pp. 2257-2269, Sep. 2003.

[2] R.G. Lorenz, and S.P. Boyd, "Robust minimum variance beamforming," IEEE Tran. Signal Processing, vol. 53, no. 5, pp. 1684-1696, May 2005.

[3] E.A. Gharavol, Y.-C. Liang, and K. Mouthaan, "Robust downlink beamforming in multiuser MISO cognitive radio networks with imperfect channel-state information," IEEE Tran. Vehicular Technology, vol. 59, no. 6, pp. 2852-2860, Jul. 2010.

[4] H. Chen, A.B. Gershman, and S. Shahbazpanahi, "Filter-and-forward distributed beamforming in relay networks with frequency selective fading," IEEE Tran. on Signal Processing, vol. 58, no. 3, pp. 1251-1262, Mar. 2010.

[5] V. Havary-Nassab, S. Shahbazpanahi, and A. Grami, "Joint receivetransmit beamforming for multi-antenna relaying schemes," IEEE Tran. Signal Processing, vol. 58, no. 9, pp. 4966-4972, Sep. 2010.

[6] Y. Hua, "An overview of beamforming and power allocation for MIMO relays," Proc. Military Commnications Conf., pp. 375-380, Oct. 2010.

[7] P. Ubaidulla, and A. Chockalingam, "Robust distributed beamforming for wireless relay networks," Proc. IEEE Personal, Indoor and Mobile Radio Communications (PIMRC) Symp., pp. 2345-2349, Sep. 2009.

[8] B. Zhang, Z. He, K. Niu, and L. Zhang, "Robust linear beamforming for MIMO relay broadcast channel with limited feedback," IEEE Signal Processing Letters, vol. 17, no. 2, pp. 209-212, Feb. 2010.

[9] E.A. Gharavol, Y.-C. Liang, and K. Mouthaan, "Robust linear beamforming for MIMO relay with imperfect Channel State Information," Proc. IEEE Personal Indoor and Mobile Radio Communications (PIMRC) Symp., pp. 510-514, Sep. 2010.

[10] C. Xing, S. Ma, Y.-C. Wu, and T.-S. Ng, "Robust beamforming for amplify-and-forward MIMO relay systems based on quadratic matrix programming," Proc. IEEE Acoustics Speech and Signal Processing (ICASSP) Conf., pp. 3250-3253, Mar. 2010.

[11] D. Ponukumati, F. Gao, and L. Fan, "Robust general rank precoding design for amplify-and-forward relay network," Proc. IEEE Global Telecommunications (GLOBCOM) Conf., pp. 1-5, Dec. 2010.

[12] K. B. Petersen and M. S. Pedersen, The Matrix Cookbook, Technical University of Denmark, Oct. 2008.

[13] I.R. Petersen, "A stabilization algorithm for a class of uncertain linear systems," Systems \& Control Letters, no. 8, pp. 351-357, 1987.

[14] M.V. Khlebnikov, and P.S. Shcherbakov, "Petersens lemma on matrix uncertainty and its generalizations," Automation and Remote Control, vol. 69, no. 11, pp. 19321945, 2008.

[15] Y.C. Eldar, A. Ben-Tal, and A. Nemirovski, "Robust mean-squared Error estimation in the presence of model uncertainties," IEEE Tran. Signal Processing, vol. 53, no. 1, Jan. 2005. 